\title{
Beneficial effects of diltiazem in coronary artery disease
}

\author{
JOHN KENNY, KIERAN DALY, GEOFFREY BERGMAN, STEVEN KERKEZ, \\ DAVID E JEWITT
}

From the Cardiac Department, King's College Hospital, London

SUMMARY The effects of the new calcium channel blocking agent diltiazem were evaluated in 11 patients with stable angina pectoris and confirmed obstructive coronary artery disease at rest and during rapid atrial pacing. Symptomatic, metabolic, coronary, and systemic haemodynamic indices were monitored at rest and during pacing induced ischaemia. At rest, after the administration of intravenous diltiazem, potent vasodilator effects were observed with a significant fall in mean blood pressure and an increase in cardiac index. Importantly, however, the systemic vasodilator effect of diltiazem was associated with no significant increase in heart rate. During pacing there was similar decrease in mean blood pressure after diltiazem, and mean pacing time to angina increased significantly. Three patients did not develop angina on repeat pacing. Coupled with an improved pacing time to angina there was a significant improvement in myocardial lactate extraction during pacing, changing from lactate production to lactate extraction after diltiazem.

This study confirms the antianginal effects of diltiazem and suggests that this agent may have advantages in the management of angina pectoris. The results suggest that diltiazem may exert its beneficial effect not only by reducing afterload reduction but also by a direct metabolic effect on the myocardium.

The calcium channel blocking agents are a heterogeneous group of drugs having in common the ability to reduce transmembrane transport of calcium ions in a variety of tissues. They differ in chemical structure and in pharmacological activity. ${ }^{1}$ Clinical interest, however, centres round their value in the management of ischaemic heart disease and cardiac arrhythmias. In angina pectoris due to obstructive coronary artery disease conventional medical treatment is directed towards reducing myocardial oxygen demand and improving myocardial oxygen supply. Calcium channel blocking agents may reduce myocardial oxygen demand by afterload reduction through peripheral vasodilatation and by reducing myocardial contractility. ${ }^{2}$ They may also improve myocardial oxygen supply through direct coronary vasodilatation.

Verapamil, a phenylalkylamine derivative, and nifedipine, a dihydropyridine derivative, have quite different pharmacological properties but are well established in the management of angina pectoris. ${ }^{34}$

Requests for reprints to Dr J Kenny, Cardiac Department, King's College Hospital, Denmark Hill, London SE5 9RS.

Accepted for publication 13 March 1984
Diltiazem, a benzothiazepine derivative, is a new addition to the calcium channel blocking agents. 5 Studies in dogs with artificially induced coronary arterial stenoses subjected to rapid cardiac pacing have indicated that diltiazem exerts a protective effect on the ischaemic myocardium by promoting a rapid recovery from ischaemia. ${ }^{6}$ Further animal studies have shown that it has a less negatively inotropic effect than either verapamil or nifedipine. ${ }^{78}$ Although in humans diltiazem improves exercise tolerance, its mechanism of action has not been clearly defined. ${ }^{910}$ We studied the effect of diltiazem on systemic and coronary haemodynamic indices and myocardial metabolism at rest and during ischaemia induced by rapid atrial pacing in patients with coronary artery disease.

\section{Patients and methods}

Eleven patients ( 10 men, one woman; mean age 55 years) with stable angina pectoris and angiographically confirmed coronary artery disease were studied. Patients with valvular heart disease, diabetes, and evidence of renal or hepatic impairment were excluded from the study. Six patients had a previous history of transmural myocardial infarction. All 
patients, however, had an ejection fraction of $>50 \%$ and a left ventricular end diastolic pressure of $<15$ $\mathrm{mm} \mathrm{Hg}$ at left ventricular angiography. In reporting the coronary arteriogram, stenoses of the luminal diameter $\geqslant 75 \%$ were considered to be significant. Seven patients had stenoses in three major vessels, one had two vessel disease, and the remaining three had single vessel disease. Antianginal medication, including specifically beta adrenergic blockade, was withdrawn for at least 72 hours before the study apart from sublingual glyceryl trinitrate, which was taken as required. Written informed consent was obtained in each case and approval for the study was obtained from the hospital ethical committee.

\section{ATRIAL PACING}

Under local anaesthesia catheters were introduced into the coronary sinus and pulmonary artery percutaneously via the left subclavian vein, and a 19 gauge needle was positioned in the femoral artery. Haemodynamic measurements of coronary sinus blood flow (thermodilution), ${ }^{11}$ cardiac output (thermodilution), and right atrial and pulmonary artery pressure were recorded. Blood samples from the coronary sinus and femoral artery were obtained simultaneously and later analysed for myocardial metabolites as previously described. ${ }^{12}$ Thirty minutes after the insertion of catheters atrial pacing was started and rapidly increased to $85 \%$ of the maximum predicted heart rate for that patient. At the onset of angina haemodynamic and metabolic measurements were made and pacing discontinued. After a rest period of 40 minutes resting haemodynamic and metabolic measurements were made and diltiazem was then administered intravenously in a bolus dose of $0.25 \mathrm{mg} / \mathrm{kg}$ over two minutes followed by a con- tinuous infusion of $0.005 \mathrm{mg} / \mathrm{kg} / \mathrm{min}$. Ten minutes later resting haemodynamic and metabolic measurements were repeated and atrial pacing was then started at the same rate as used during the control pacing period. At the point equivalent in pacing time to the onset of angina during the control pacing period haemodynamic and metabolic measurements were repeated. If angina had not occurred pacing was continued to the new onset of angina or until 13 minutes had elapsed.

\section{CALCULATIONS}

Myocardial extraction ratios (ER) for specific substrates were calculated as $E R=A-V / A \times 100$ where $A$ and $V$ are arterial and venous substrate concentrations respectively. The extraction ratio was expressed as a percentage. Myocardial oxygen consumption was derived as (arterial oxygen content minus coronary sinus oxygen content) $\times$ coronary blood flow, and expressed as $\mathrm{ml} / \mathrm{min}$. Statistical analysis of the resting and paced variables was performed using Student's paired $t$ test.

\section{Results}

The Table shows the haemodynamic and metabolic effects of diltiazem at rest and during pacing.

\section{HAEMODYNAMIC AND METABOLIC EFFECTS \\ At rest}

At rest diltiazem caused systemic vasodilatation with significant falls in mean (SEM) arterial pressure and total systemic vascular resistance $(19.0$ (1.7) $v 14.6$ (1.3) units, $\mathrm{p}<0.001)$. Vasodilatation was accompanied by an increase in cardiac index $(2.9(0.2) v 3.4$ $\left.(0.3) \mathrm{l} / \mathrm{min} / \mathrm{m}^{2}, \mathrm{p}<0.02\right)$ and stroke volume index.

Table Haemodynamic and metabolic indices before and after diliazem at rest and during atrial pacing. Values are means (SEM)

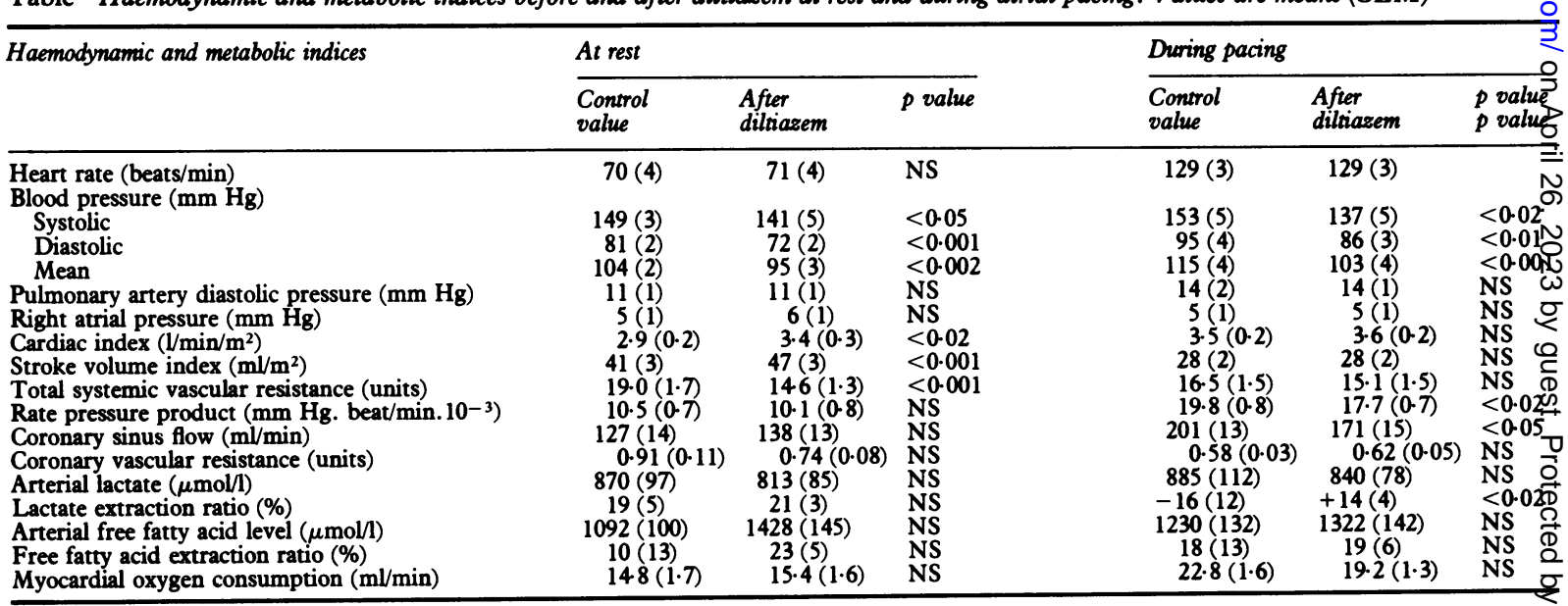


Despite the fall in systemic vascular resistance, however, there was no significant change in resting heart rate $(70$ (4) $v 71$ (4) beats/min) after diltiazem. Pulmonary artery diastolic and right atrial pressures were not significantly altered. Although diltiazem produced a small increase in coronary sinus flow and a reduction in coronary vascular resistance, these changes did not reach statistical significance. At rest there was no significant alteration in metabolic indices after diltiazem.

\section{During pacing}

During pacing diltiazem again produced a significant fall in mean arterial pressure and a concomitant reduction in rate pressure product $(19.8(0.8) v 17.7$ (0.7) $\mathrm{mm} \mathrm{Hg}$. beats/min. $\left.10^{-3}, \mathrm{p}<0.02\right)$. In contrast to the findings at rest, however, there were no significant changes in cardiac index, stroke volume index, or total systemic vascular resistance. There was a small but significant decrease in coronary sinus blood flow during pacing. After diltiazem there was a significant change in lactate extraction ratio from mean (SEM) lactate production of $-16(12) \%$ to mean lactate extraction of $+14(4) \%(p<0.02)$. There were no significant changes in arterial lactate or arterial free fatty acid concentation or in free fatty acid extraction

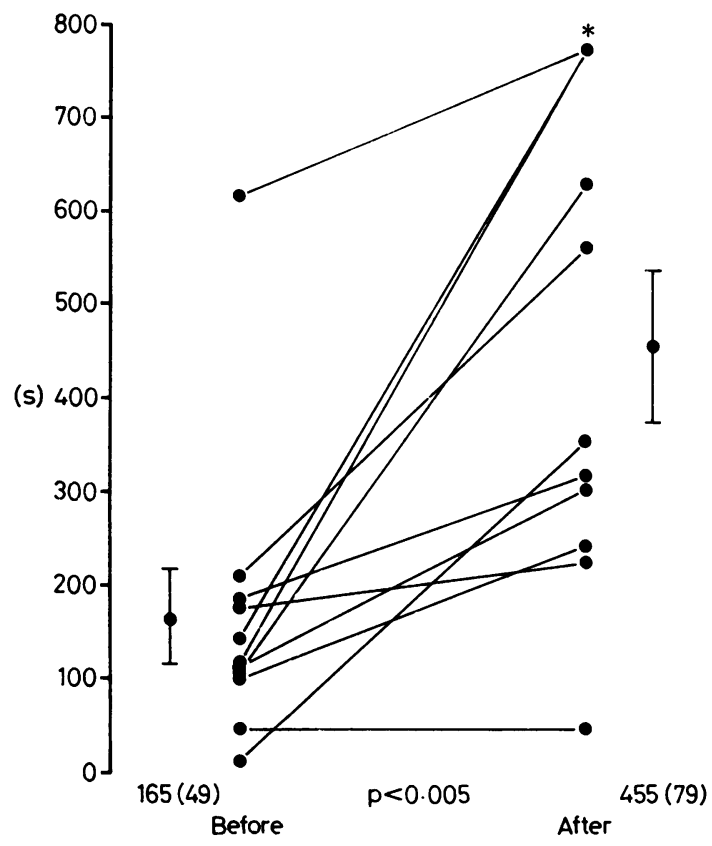

Figure Individual and mean (SEM) pacing times to angina (S) before and after intravenous administration of diltiazem. *Denotes three patients who did not develop angina on repeat pacing. ratios. Although myocardial oxygen consumption was reduced after diltiazem, this change was not statistically significant.

\section{PACING TIME TO ANGINA}

After the administration of diltiazem angina did not occur in three patients on repeat pacing. In the remaining patients, with the exception of one whose pacing time to angina remained virtually unchanged, the onset of angina was delayed. Consequently mean pacing time to angina after diltiazem was significantly increased from 165 (49) to $455(79) s(p<0.005)$ (Figure).

\section{Discussion}

The haemodynamic findings at rest in this study indicate the potent vasodilator effect of diltiazem. A study in dogs using intracoronary diltiazem showed it to have a negative inotropic effect, although this was less than that produced by equimolar doses of nifedipine or verapamil. ${ }^{8}$ In this study cardiac output at rest increased significantly after administration of the drug, and there were no significant changes in pulmonary artery diastolic pressure suggesting the absence of negative inotropy. Any negative inotropic effect might, however, have been offset by the positive inotropic effect of increased sympathetic discharge in response to systemic vasodilatation.

In this study systemic vasodilatation occurred without any significant change in resting heart rate. This is in contrast to an increase in resting heart rate seen after acutely administered nifedipine. ${ }^{13}$ The absence of heart rate response to systemic vasodilatation after diltiazem has been reported in an exercise study of long term oral administration. ${ }^{9}$ In addition, electrophysiological studies have shown that diltiazem depresses sinoatrial node automaticity, and this may in part account for its effect on heart rate. ${ }^{14}$ This absence of an increase in heart rate and thus in myocardial oxygen demand may have important advantages in the management of angina pectoris.

In ischaemic heart disease angina may be induced by rapid atrial pacing and is associated with a change from aerobic to anaerobic metabolism. The reproducibility of pacing time to angina and the degree of change in lactate metabolism are well established and thus each patient may be reliably used as his own control. ${ }^{15}$ In this study the beneficial effect of diltiazem in angina pectoris was shown by a significant prolongation of mean pacing time to angina concomitant with an improvement in myocardial lactate extraction. Such a beneficial effect in stable angina pectoris may have been multifactorial in origin. Calcium channel blocking agents may increase myocardial oxygen supply and decrease myocardial oxygen 
demand. In addition, by inhibiting the rise in myocardial calcium induced by ischaemia they may prevent or delay the consequences of ischaemia. ${ }^{2}$ In this study diltiazem did not produce any significant change in resting coronary sinus blood flow but resulted in a small but significant decrease during pacing. A similar effect has been reported with verapamil, which, although reducing coronary sinus flow at rest and during pacing, prolonged pacing time to angina and improved myocardial metabolism. ${ }^{16}$ This would suggest that the antianginal effect is not due to increased coronary blood flow. In animal studies, however, under conditions simulating unstable angina pectoris, diltiazem increased collateral blood flow to collateral dependent myocardium. ${ }^{17}$ We cannot exclude the possibility in this study that diltiazem caused a redistribution of the available blood supply by increasing collateral flow.

During pacing diltiazem reduced left ventricular afterload and rate pressure product. This might be expected to reduce myocardial oxygen consumption, as rate pressure product during pacing induced tachycardia is a good indicator of the level of myocardial oxygen demand. ${ }^{18}$ Surprisingly, in this study we failed to show a significant reduction in myocardial oxygen consumption during pacing, although a trend towards reduction was seen. This suggests that diltiazem may have had a direct metabolic effect on the myocardium. The calcium antagonist nifedipine has been shown to have an antianginal effect unrelated to its effect on systemic and coronary haemodynamics indices, ${ }^{19}$ perhaps through inhibition of calcium ion flux at a cellular level. In a similar way, diltiazem may have exerted a direct effect on the myocardium so delaying the onset or reducing the consequences of ischaemia. Our study design, however, did not permit evaluation of a possible beneficial effect at cellular level.

In conclusion, this study showed the potent vasodilator effect of diltiazem, which occurred without a significant change in resting heart rate. The beneficial effect in angina pectoris is indicated by an increased pacing time to angina and an improvement in myocardial metabolism. The results suggest that diltiazem acts by a combination of effects, such as afterload reduction and a direct effect in the myocardium. Diltiazem may have certain advantages over other presently available calcium channel blocking agents. It can be given both orally and intravenously, and a multicentre study to assess drug safety showed a low incidence of side effects. ${ }^{10}$ Comparisons between diltiazem and other calcium channel blocking agents would be of interest. The results of this study indicate that diltiazem may well prove to be a very useful addition to the present treatment of angina pectoris.

\section{References}

1 Henry PD. Comparative pharmacology of calcium antagonists: Nifedipine, Verapamil and Diltiazem. Am $\mathcal{f}$ Cardiol 1980; 46: 1047-58.

2 Braunwald E. Mechanism of action of calcium-channel-blocking agents. N Engl f Med 1982; 307: 1618-27.

3 Balasubramanian V, Paramasivan R, Lahiri A, Raftery EB. Verapamil in chronic stable angina. Lancet 1980; i: 841-4.

4 Lynch P, Dargie H, Krikler S, Krikler D. Objective assessment of antianginal treatment: a double-blind comparison of propranolol, nifedipine and their combination. $\mathrm{Br} \operatorname{Med} \mathcal{F} 1980 ; 281$ : $184-7$.

5 Sato M, Nagao T, Yamaguchi I, Nakajimi H, Kiyomoto A. Pharmacological studies on a new 1,5 -benzothiazepine derivative $($ CRD-401 $=$ Diltiazem $)$. I. Cardiovascular actions. Arzmeim Forsch 1971; 21: 1338-43.

6 Sasayama S, Takahashi M, Nakamura M, et al. Effect of diltiazem on pacing-induced ischemia in conscious dogs with coronary stenosis: improvement of postpacing deterioration of ischemic myocardial function. Am $\mathcal{F}$ Cardiol 1981; 48: 460-7.

7 Millard RW, Lathrop DA, Grupp G, Ashraf M, Grupp IL, Schwartz A. Differential cardiovascular effects of calcium channel blocking agents: potential mechanisms. Am $\mathcal{J}$ Cardiol 1982; 49: 499-506.

8 Walsh RA, Badke FR, O'Rourke RA. Differential effects of systemic and intracoronary calcium channel blocking agents on global and regional left ventricular function in conscious dogs. Am Heart f 1981; 102: 341-50.

9 Hossack KF, Bruce RA, Trimble S, Kusumi F. Improved exercise performance in patients with stable angina pectoris receiving diltiazem. Am f Cardiol 1981; 47: 95-101.

10 Strauss WE, McIntyre KM, Parisi AF, Shapiro W. Safety and efficacy of diltiazem hydrochloride for the treatment of stable angina pectoris: report of a cooperative clinical trial. Am $\mathcal{f}$ Cardiol 1982; 49: 560-6.

11 Ganz W, Tamura K, Marcus HS, Donoso R, Yoshida S, Swan HJC. Measurement of coronary sinus blood flow by continuous thermodilution in man. Circulation 1971; 44: 181-95.

12 Bergman G, Atkinson L, Metcalfe J, Jackson N, Jewitt DE. Beneficial effect of enhanced myocardial carbohydrate utilisation after Oxfenicine in angina pectoris. Eur Heart $\mathcal{f}$ 1980; 1: 247-53.

13 Stone DL, Stephens JD, Banim SO. Coronary haemodynamic effects of nifedipine: comparison with glyceryl trinitrate. $\mathrm{Br}$ Heart f 1983; 49: 442-6.

14 Kawai C, Konishi T, Matasuyama E, Okazaki H. Comparative effects of three calcium antagonists, diltiazem, verapamil and nifedipine, on the sinoatrial and atrioventricular nodes. Circulation 1981; 63: 1035-42.

15 Jackson G, Atkinson L, Oram S. Improvement of myocardial metabolism in coronary arterial disease by beta-blockade. $\mathrm{Br}$ Heart f 1977; 39: 829-33.

16 Ferlinz J, Turbow ME. Antianginal and myocardial metabolic properties of verapamil in coronary artery disease. Am $\mathcal{F}$ Cardiol 1980; 46: 1019-26.

17 Franklin D, Millard RW, Nagao T. Responses of coronary collateral flow and dependant myocardial mechanical function to the calcium antagonist, diltiazem. Chest 1980; 78 (suppl): 200-4.

18 Holmberg S, Varnauskas E. Coronary circulation during pacinginduced tachycardia. Acta Med Scand 1971; 190: 481-90.

19 Kaltenback M, Schulz W, Kober G. Effects of nifedipine after intravenous and intracoronary administration. Am $\mathcal{f}$ Cardiol 1979 ; 44: 832-8. 